\title{
A Method for Shot Boundary Detection and R-Frame Selection of Digital Video
}

\author{
J. Y. Lee, S. Y. Jeong, B. T. Chun, Y. J. Bae \\ Image Processing Division, Systems Engineering Research \\ Institute \\ PO Box 1, Yusung-gu, Taejon, KOREA, 305-600 \\ Tel)+82-42-869-1454, Fax) +82-42-869-1479, \\ e-mail)leejy@seri.re.kr
}

\begin{abstract}
For automated analysis of video contents and efficient browsing, it is essential to extract the internal structure of the video contents. For this purpose, three technologies (a robust shot boundary detection algorithm, an R-frame selection algorithm, an editor that allows manual edition of the structure of the video contents to complement the first two automated processes) are needed. This paper addresses the first two problems.

For shot boundary detection, a new algorithm including dual detectors for the two types of boundaries, cuts and gradual boundaries, is proposed. Also, some peculiar shot boundaries that are reported from each detector could be eliminated by adopting a delayed decision mechanism rather than immediate decision at each detector level. Therefore, the proposed algorithm produces high accuracy of 0.974 in recall and 0.942 in precision, which are far better results than those of the existing algorithms compared.

For R-frame selection, the changing pattern of the pixel differences is investigated. The existing algorithms mainly adopt simple mechanical approaches such as a fixed period sampling. By contrast, the proposed approach considers the motion in contents by using pixel differences, and thus, can select more meaningful frames. Furthermore, since the proposed algorithm reuses the pixel differences which is already calculated for the shot boundary detection, the total processing time is not much increased.
\end{abstract}

Keywords

Digital Video, Shot Boundary Detection, Dual Detector, R-frame

Visual Database Systems 4 Y. Ioannidis \& W. Klas (Eds.)

C) 1998 IFIP. Published by Chapman \& Hall 


\section{INTRODUCTION}

For automated analysis of video contents and efficient browsing, it is essential to extract the internal structure of the video contents. For example, a news video generally consists of several sections such as politics, economics, sports, etc. Each section is also comprised of several topics. Using this hierarchical structure information, users can far more efficiently access the necessary information. On the basis of this consideration, hierarchical data models (Huang, 1996) (Zhong, 1996) (Zhang, 1995) as shown in Fig. 1 have been proposed. In the figure, the sequence levels can be mapped to sections in a news video, while scene levels correspond to individual topics. The shot level is generally defined as an unbroken sequence of frames from one camera (Boreczky,1996). The R-frames (Representative frames) are the frames that can stand for the contents of the shot.

In this hierarchical model, sequence and scene levels should be determined on the basis of the high-level semantic information, however, it is very difficult to automatically extract with the current state of the art. On the contrary, shot boundary detection is relatively feasible. This is because the difference between the prior and the posterior frames of the boundary is large in general. Hence, it is reasonable to detect shot boundaries, and to select R-frames automatically and to construct the scene and sequence levels by manually combining the detected shots.

Therefore, the following three technologies are required to be developed in order to extract internal structure of video contents.

1) A robust and reliable shot boundary detection algorithm

2) An R-frame selection algorithm that can select meaningful frames from the shot

3) A video description editor that allows manual combination of the shots into scene or sequence levels

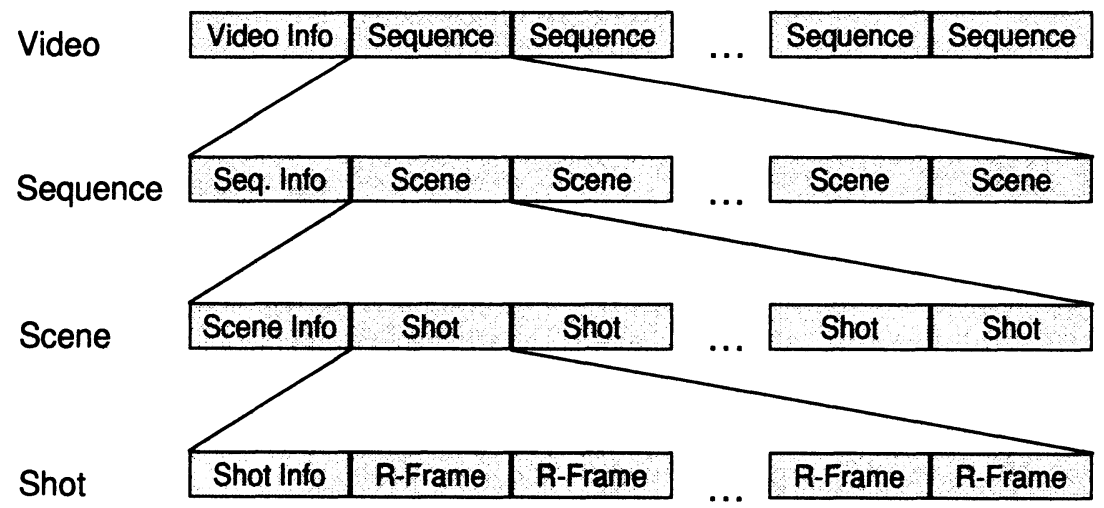

Fig. 1. Internal Structure of Video Contents 

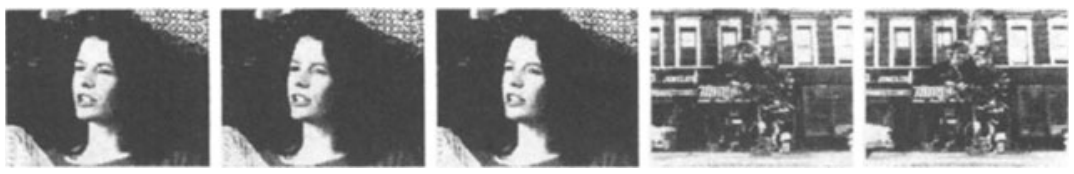

Fig. 2. An Example of Cuts
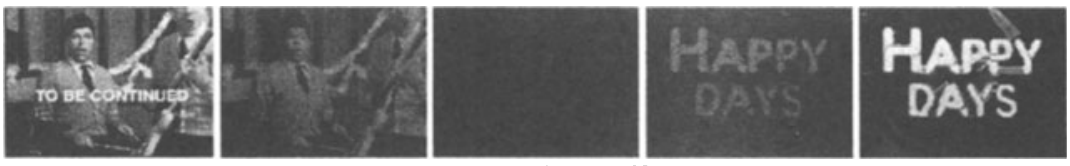

(a) Fading Effects
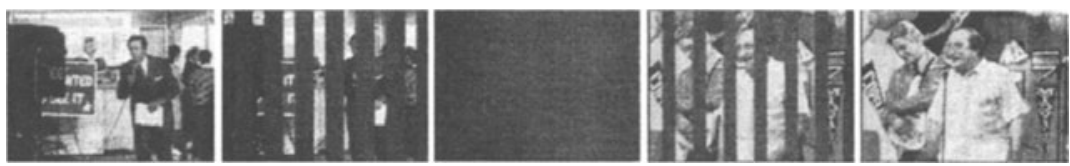

(b) Curtaining Effects
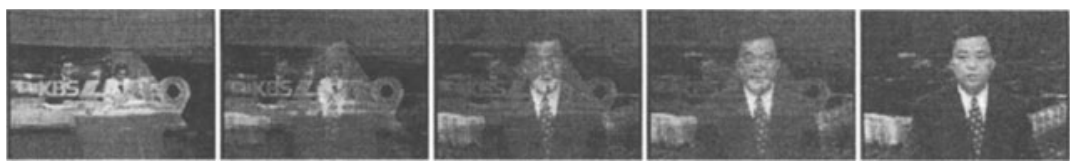

(c) Dissolve and Merge
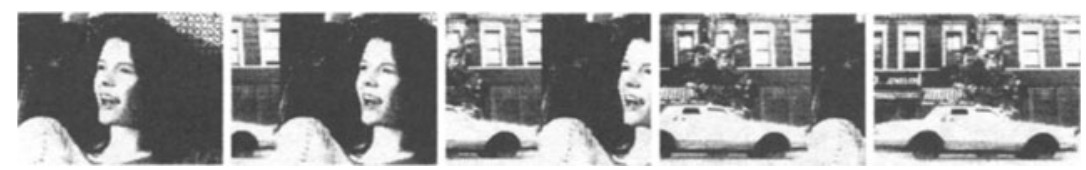

(d) Wiping Effects

Fig. 3. Types of Gradual Boundaries

This paper addresses the first two problems.

In general, the shot boundaries can be classified into two types (Ahanger, 1996) (Zhang, 1994). The first one is called as a cut that is an abrupt shot change in one frame as shown in Fig. 2. Many boundaries belong to this type.

In these boundaries, the prior $\left(3^{\text {rd }}\right)$ and the posterior $\left(4^{\text {th }}\right)$ frames of the boundary show very different characteristics in terms of their contents. Hence, in case of a cut boundary, relatively good results can be obtained by simply calculating pixel differences between consecutive frames. Another one is a gradual boundary that is generated in the process of video editing such as fading, curtaining, dissolve and merge or wipings as shown in Fig. 3. In this type of boundaries, the difference between consecutive frames is not so large that other than the difference 
measure should be considered for the detection.

This paper proposes a new shot boundary detection algorithm including dual detectors for the above two types of boundaries. In order to detect cuts, a new feature, variation of the difference histogram, is proposed, which has been proved to be very effective. Also by combining the new feature with the traditional features using a neural network, the accuracy in cut detection is greatly improved. For the gradual shot boundaries, a subset of them where a solid color frame passes by in transiting to the next shot as in the case of fading or curtaining, is considered. By observing the appearance of a solid color frame by the pixel variance of each frame that decreases rapidly when fading out is occurring, this type of gradual boundaries could be reliably detected.

The field of R-frames selection methods has attracted relatively less attention until now, and thus simple mechanical approaches such as a fixed period sampling have been adopted (Ardizzone, 1996). However, in considering image content based searching, the image features are generally extracted from R-frames only. Thus if the selected $R$-frame is not a representative one as the name implies, a search may be failed. Also R-frames are important as visual indices to the shot in browsing digital video. Considering the importance of $\mathrm{R}$-frame, there should be a more sophisticated algorithm.

This paper proposes a selection algorithm of using the pattern of changes in pixel differences, which can select more meaningful frames than the existing mechanical approaches. Also, the increase in processing time can be negligible since the pixel differences produced from shot boundary detection are reused.

\section{RELATED WORKS}

The most intensively investigated topic in shot boundary detection is to find out a robust feature that can detect cuts without being confused by the fast motion, camera operation or sudden brightness changes. The most intuitive one may be the approach that uses the pair-wise pixel differences between consecutive frames (Boreczky, 1996). If the difference is greater than a predetermined threshold, a boundary is declared. However this approach is valid only when the content is relatively static. If a large object is moving or fast camera operation is occurring, false boundaries may be declared.

To compensate this weakness to motion, other methods that use histogram difference are proposed (Ahanger, 1996) with the assumption that the continuous frames may have similar image characteristics even though the details are changed due to motion. Contrary to using pixel differences, these approaches tend to miss boundaries if shots of similar atmosphere appear in succession.

Some researchers have paid attention to edge information (Ahanger, 1996). Edge conveys the information about the objects included in the frame rather than overall characteristics of the image, thus more reliable detection is expected in theory. However the experimental results have not been so satisfactory and this approach has a serious demerit in processing time. In shot boundary detection, the 
processing time is one of the most important factors that should be considered because generally the size of digital video is extremely large.

Also there are approaches based on motion analysis (Boreczky, 1996). The idea is that if the consecutive frames are continuing ones, there should exist resemblance between them and consistent motion factor or camera operation can be detected. This approach also has demerits in processing time because the motion analysis generally requires block matching that is computationally expensive. From this respect, there have been proposed algorithms that utilize the motion vectors included in MPEG encoded digital video.

By using the motion vector already extracted in the encoding process, motion analysis could be far faster. Further it is more advantageous because the timeconsuming decoding process is not required. Considering that most of the digital video is provided in compressed form, the significance of analyzing digital video without decoding is very large. In this area, there have been reported the algorithms that detect shot boundaries by using the difference of DCT coefficients extracted directly from MPEG encoded files (Liu, 1995) (Meng, 1996) (Zhang, 1996). Even though these approaches have not been successful especially in accuracy (Boreczky, 1996), it is still a hot issue that should be investigated.

Another issue in automatic shot boundary detection is the method of gradual boundary detection. Compared to the cut detection, the researches on this topic have been less intensive. It is partly due to the fact that gradual boundaries are far scarcer than cuts so that the development of a robust cut detection algorithm was more important in practical sense. Another reason is that the detection of gradual boundaries is more difficult because their types are so diverse.

In this research topic, a dual threshold method proposed by Zhang et al may be the representative one (Zhang, 1996) (Ardizzone, 1996). This method adopts two thresholds. One is a high threshold that declares shot boundary if that threshold is exceeded. Another is a low threshold that initiates accumulating the histogram differences. This accumulation continues until the accumulated sum exceeds the high threshold, where a shot boundary is declared. However this approach has a risk of declaring false boundaries when the histogram difference becomes large due to the fast motion of big objects in the contents.

Another important topic in extracting the internal structure of video content is the selection method of $\mathrm{R}$-frames. In the previously reported methods for automated video content analysis, the selection of $\mathrm{R}$-frames are relatively ignored and mainly simple methods such as selecting a frame every other second or selecting the first and the last frame are adopted (Carcia, 1996) (Picard, 1995). Although an improved approach that can control the density of R-frames by user adjusted parameters (Zhang, 1995) was reported, more sophisticated algorithm is still expected considering the importance of $\mathrm{R}$-frames in searching and browsing.

$R$-frames are the frames that stand for the semantic information of the shot. Hence for the reasonable selection, general understanding of the shot contents should be assumed in principle, which is not yet possible. To avoid the problem, some rules of thumb are thought to be necessary that can select acceptable frames. 


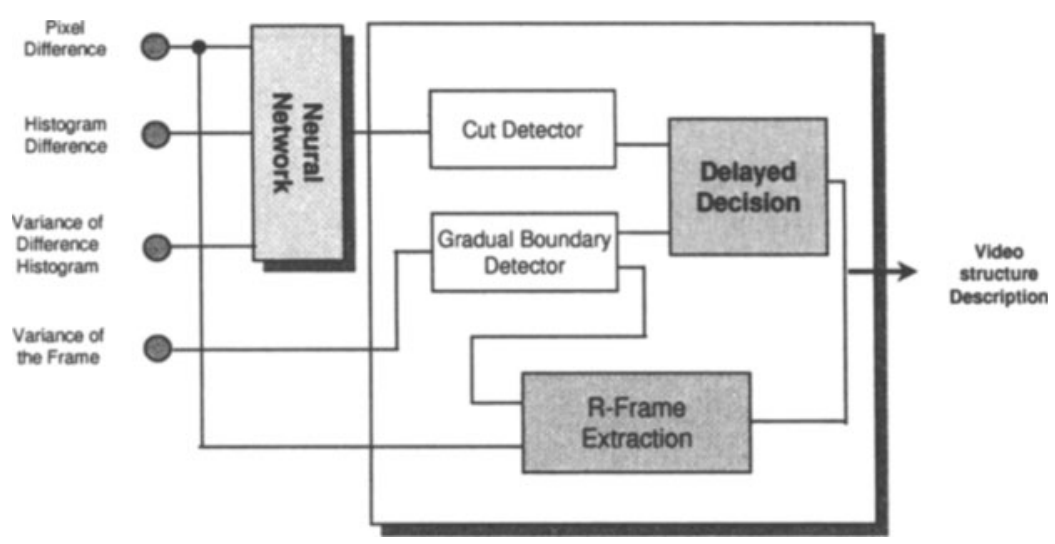

Fig. 4. The Proposed Algorithm

\section{THE PROPOSED ALGORITHM}

The flow of the proposed algorithm is shown in Fig. 4. It has dual detectors that are specifically designed to detect cuts and gradual boundaries, respectively, contrary to the existing algorithms that have tried to detect two kinds of boundaries with a single mechanism. Each detector observes the video stream carefully based on its own decision mechanism. However instead of declaring boundary by themselves, their observation results are reported to the delayed decision module.

The delayed decision module has two roles. One is to arbitrate the results of the two independent detectors if some conflicts exist. Another is a kind of postprocessing that determines if the reported candidate of boundary is reasonable.

The R-frame selector selects one or several frames that can stand for the contents of the shot. The number of selected R-frames is determined by analyzing the contents. In static shots that consist of almost identical frames, only one frame is selected, while several frames are selected in a dynamic shot. For this decision, motion information is of great help. However in the proposed algorithm, instead of executing intensive motion analysis that requires a lot of processing, pixel differences already calculated in the shot boundary detection process are reused.

\subsection{Cut Detector}

\subsubsection{A Variance of Difference Histogram}

In continuing frames, the pair-wise pixel differences between consecutive frames tend to crowd in low values as in Fig. 5 (a). On the contrary, if cut exists, two consecutive frames may have no correlation, which results in flat distribution in difference histogram as shown in Fig. 5 (b).

Hence the variance of the difference histogram that is calculated by the 


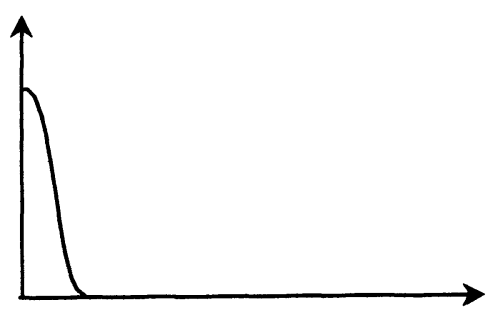

(a) at continuing frames

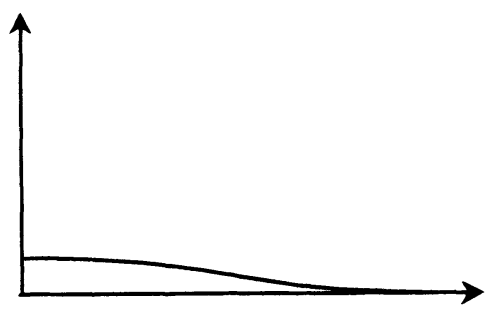

(b) at shot boundaries

Fig. 5. Characteristics of Difference Histogram

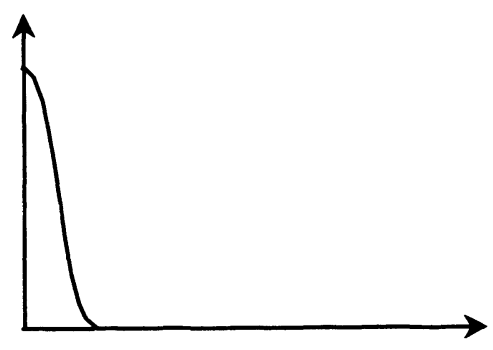

(a) no brightness change

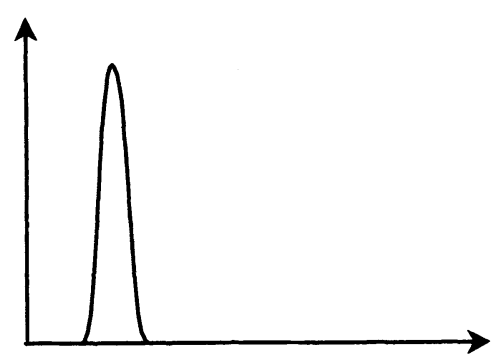

(b) brightness changed

Fig. 6. Difference Histogram when Brightness Changes

following formula becomes very small if cut exists.

$$
D=\frac{\sum_{i=1}^{K}\left(H_{m, m+1}(i)-H\right)^{2}}{K}
$$

(Here, $\mathrm{H}_{\mathrm{m}, \mathrm{m}+1}$ is the difference histogram calculated between $\mathrm{m}$-th and $(\mathrm{m}+1)$-th frame, $\mathrm{K}$ is the range of the difference values, and $\mathrm{H}$ is the mean of $\left.\mathrm{H}_{\mathrm{m}, \mathrm{m}+1}\right)$.

This metric is especially robust against brightness changes of the frames. When the brightness suddenly changes, the difference histogram is shifted from Fig. 6 (a) to Fig. 6 (b) of the figure, but the variance value is not much influenced.

\subsubsection{Combining Multiple Features}

In general, the features that have been proposed by many researchers including the one proposed here have different advantages in different situations. For example, histogram difference is relatively robust against large motions but often fails to detect the boundaries of shots that have similar image characteristics, while pixel 
difference is good at detecting such cases. Hence if the features are combined appropriately, a more desirable result can be expected. Here three features, the variance of the difference histogram described in 3.1.1 and the following two features, are selected for the verification of the idea.

1) Pixel Difference

$$
D_{p}=\frac{\sum\left(\operatorname{lm}(x, y)-\ln _{\mathrm{m}+1}(x, y)\right)}{N}
$$

(Here, $I_{m}(x, y)$ is the pixel intensity of $\mathrm{m}$-th frame at coordinate $(x, y), N$ is the total number of pixels in a frame)

2) Histogram Difference

$$
D_{h}=\frac{\sum_{i=1}^{K}\left|H_{m}(i)-H_{m+1}(i)\right|}{K}
$$

(Here $\mathrm{H}_{\mathrm{m}}$ is the histogram of $\mathrm{m}$-th frame, $\mathrm{K}$ is the range of pixel values)

As a method of combining the features, there can be a lot of alternatives such as multi-level slicing, minimum distance method or maximum likelihood method (Tou, 1974). As another alternative, recently neural networks have been widely used for these purposes and have been successful in various applications. Considering these reported results and simplicity of implementation, a neural network of back error propagation model is adopted for the combination.

In Fig. 7, the structure of the adopted neural network is shown. The neural network has an input layer of three neurons that correspond to the above three features, one hidden layer, and an output layer of two neurons that correspond to the shot boundary and continuing frames respectively.

For the training of the neural network, a news video of approximately 1,500

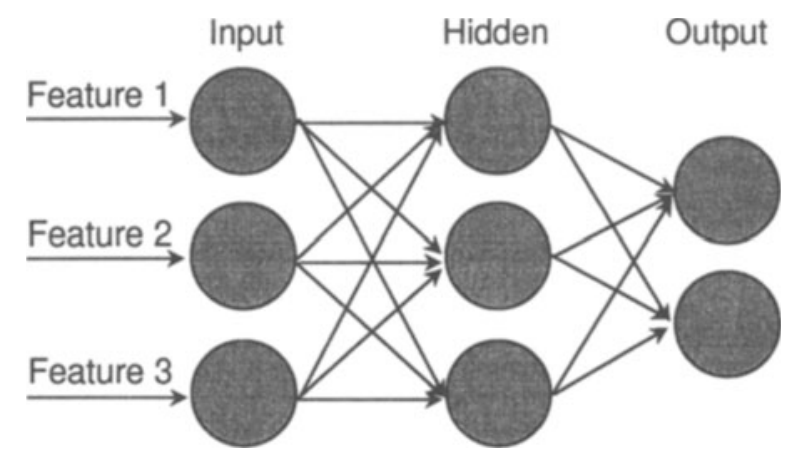

Fig. 7. Configuration of Adopted Neural Network 
frames is used. This data contains no gradual boundaries but cuts (actually 32 cuts exist). To prohibit the preponderance of training, the features for cuts are repeatedly applied to the network 50 times.

\subsection{Gradual Boundary Detector}

Among gradual boundaries, only the ones that include a solid color frame as in fading or curtaining effect are considered here. Instead of focusing on differences between consecutive frames as in a cut detection, the status of the individual frame is observed to watch if the sequence reaches a solid color frame. As a metric for the observation, a pixel variance of the frame image is adopted, which becomes very small for solid color frames.

There are two reasons why the pixel variance is adopted. The first one is that it can detect the final status of the gradual boundary (a solid color frame) very reliably, and the second one is that it can be easily computed from the histogram already obtained for cut detection, using the following formula.

$$
V=\frac{\sum_{i=1}^{K} H(i) \bullet(i-M)^{2}}{N}
$$

(where, $\mathrm{H}$ is the intensity histogram of the image, $\mathrm{K}$ is the range of pixel values, $\mathrm{N}$ is the total number of pixels and $\mathrm{M}$ is the average intensity value of the pixels which also can be easily calculated from the histogram $\mathrm{H}$ ).

When a sequence is fading out, the pixel variance may decrease rapidly and may increase again if fading in starts as shown in Fig. 8. The purpose is to declare a shot boundary at the point $S$ in the figure. For this purpose, once the variance goes down under the pre-determined threshold (shown in dotted line), the

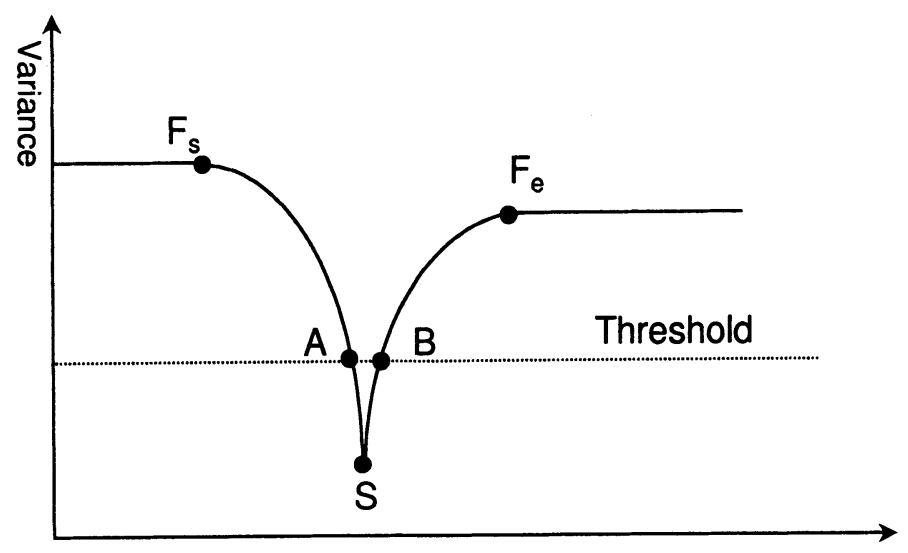

Fig. 8. Detecting Gradual Shot Boundaries 

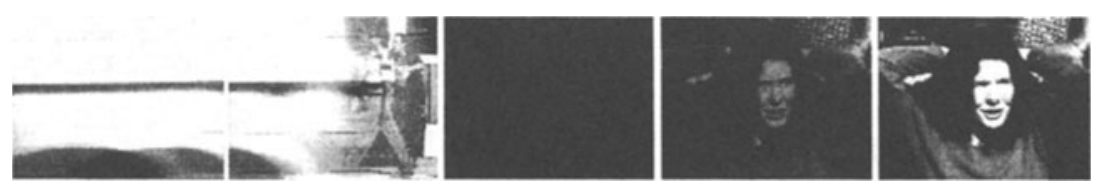

Fig. 9. Fading In without Fading Out

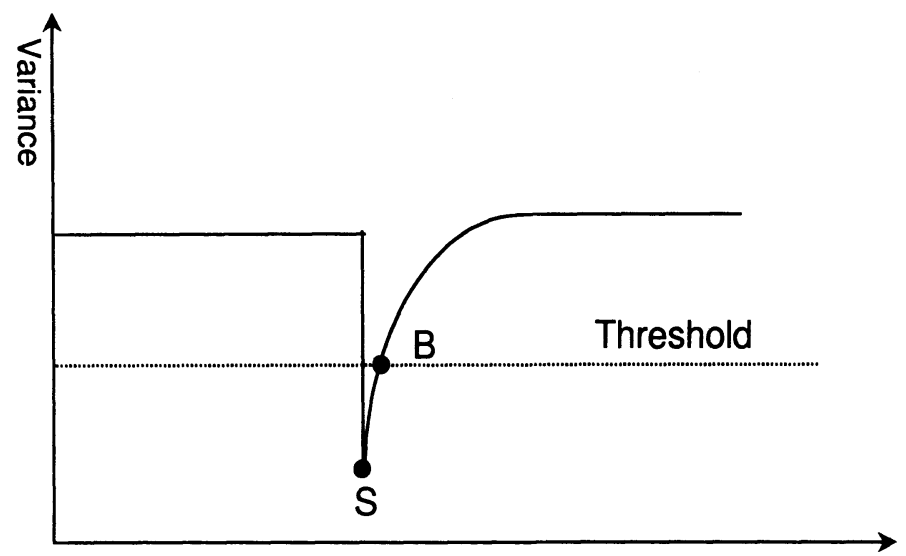

Fig. 10. Variances in case of Fig. 9.

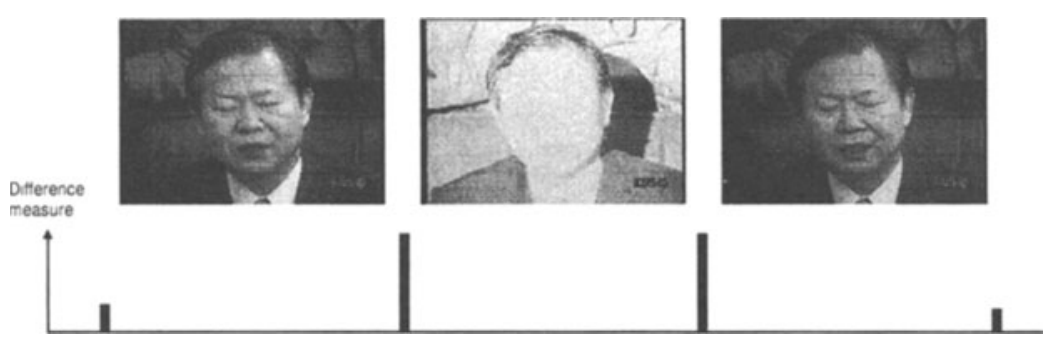

Fig. 11. Undesirable Boundaries

subsequent variances are saved in an array until the variance goes up over the threshold again. That is, the variances from the point A to the point B are saved in an array. And the minimum value in the array is searched to find the point $S$.

\subsection{Delayed Decision}

The proposed algorithm has two independent detectors, thus there are possibilities the results of the two detectors conflict. Since the two detectors are designed to 
detect different types of boundaries, it is natural that one detector reports a boundary but another reports nothing. The problem occurs when two detectors report the boundary at a similar location. Fig. 9 shows an example of such a case. Here fading in occurs without fading out. At the $3^{\text {rd }}$ frame of the sequence, both detectors report a shot boundary. In this case, the saved variance array that is reported from gradual boundary detector shows the characteristics of Fig. 10. With this information, the end of the prior shot is declared as a cut and the start of the posterior shot as a gradual boundary. As will be described below, this boundary type information is important in selecting R-frames.

Another important role of delayed decision module is to check the validity of the detected boundaries. Fig. 11 shows an example of a sudden brightness change that is caused by the camera flashes of the press reporters. In this case, the cut detector may report two consecutive frames as boundary candidates, which is apparently not desirable. To avoid the false detection like this, delayed decision module checks the length of the shot candidates (in case of Fig. 11, a shot of one frame length appears). If the length is shorter than a pre-determined threshold, delayed decision module commands the cut detector to check if the prior and the posterior frame of the shot (the $1^{\text {st }}$ and $3^{\text {rd }}$ frame of Fig.11) have sufficient difference to declare a cut. If it is the case, the frames are considered as a kind of gradual boundary and only one boundary is declared eliminating the peculiar small length frame shot. Otherwise, the reported shot boundaries are ignored (in case of Fig. 11, the boundaries are ignored). With this delayed decision mechanism, a lot of unreasonable shot boundaries could be eliminated.

\subsection{Selection of R-Frames}

If the content of the shot is very static as in an anchor shot of news videos, only one arbitrarily selected frame can sufficiently stand for the contents. On the contrary, the shot may contain frames of various objects as in the shots where the camera is continuously panning for the large area. For these shots, it is desirable to select several frames that contain mutually different contents. Also if a camera is moving very quickly as shown in Fig. 12, it is reasonable to think that the quickly moving frames are only transient states and finalized status (face of the man) is the theme of the shot.

With the above considerations, motion information seems necessary for the meaningful selection of R-frames. However instead of the time-consuming general motion analysis, the proposed algorithm uses the pixel difference that is already calculated for shot boundary detection and is known relatively sensitive to motions.

The proposed method classifies the changing pattern of the pixel differences into three types of Fig. 13, and select R-frames in the following manner for each pattern. In the figure, the black dots show where the $\mathrm{R}$-frame is selected.

Pattern A : This type is a very static shot, thus any frame can stand for the contents. In this case, the center frame of the shot is selected as an R-frame. 

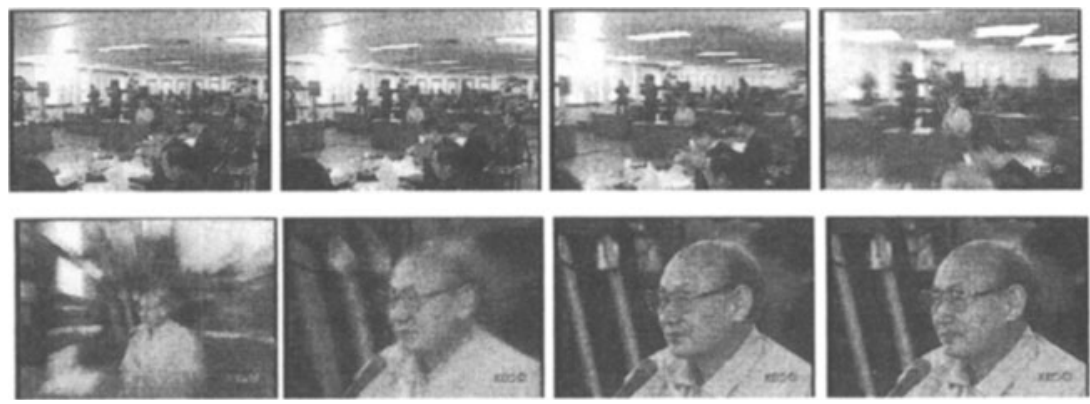

Fig. 12. Fast Zoom In

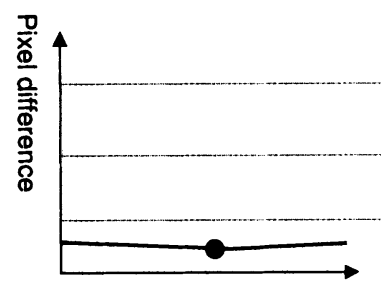

(a) pattern $\mathrm{A}$

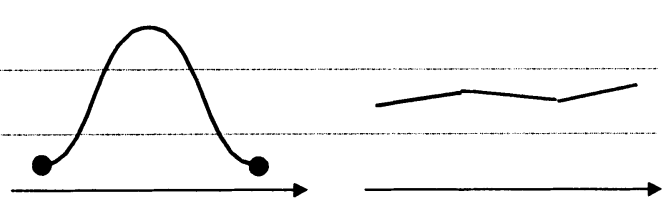

(b) pattern $B$ (c) pattern $\mathrm{C}$

Fig. 13. Changing Pattern of Pixel Differences

Pattern B : This is the case described in Fig. 12. In this type, the start and end of the transient state is selected as R-frames.

Pattern C : This type has no special clue to select an R-frame. In this case, a fixed period approach is adopted.

Also fading should be considered for the reasonable $\mathrm{R}$-frame selection because it is not desirable to select the frames in the process of fading in or fading out. For this reason, the frames between the point $F_{s}$ and the point $F_{e}$ of Fig. 8 are excluded from the target of $R$-frame selection described above.

\section{EXPERIMENT}

The proposed algorithm is tested on digital videos of MPEG and AVI format. The contents of the experimental data are news, music, animation and sports, and contain approximately 100,000 frames (55 minutes) in total. There are approximately 800 cuts and 30 gradual boundaries in the experimental data (generally, the number of gradual boundaries is far smaller than that of cuts).

For the comparison, the algorithms using the following features are implemented. 
1) A pixel difference,

2) A histogram difference,

3) An edge count difference,

4) A variance of difference histogram

5) the proposed algorithm

The algorithms are evaluated based on the following values.

$$
\begin{aligned}
& \text { Recall }=\frac{\text { Correct }}{\text { Correct }+ \text { Missed }} \\
& \text { Precision }=\frac{\text { Correct }}{\text { Correct }+ \text { FalsePositive }}
\end{aligned}
$$

Large recall values mean that the correct shot boundaries are not missed too much. Large precision values mean that few false shot boundaries are declared. Of course, these two values are interdependent and closely related to the threshold values. Here, the best result where recall plus precision becomes largest is shown.

The experimental result is shown in Table 1. As shown in the result, the proposed algorithm shows far better results than other compared algorithms. Also the variance of the difference histogram that proposed in this paper is proved to be a good feature in detecting shot boundaries. The precision and recall of the proposed algorithm corresponds to 51 false positive and 22 false negative detection in approximately 100,000 frames, which is supposed to be sufficiently practical.

The validity of R-frame selection is very difficult to evaluate quantitatively because it is a subjective problem. However for the very static shots, the proposed algorithm selected only one frame compared to the 3 to 20 frames of the existing algorithms. Also owing to excluding the gradually changing regions, no cumbersome frames are selected when a gradual boundary exist.

\section{CONCLUSION}

For the important problem of searching and browsing of digital video, the extraction of the internal structure of the video contents is very important. In this

Table 1. Comparison on Accuracy

\begin{tabular}{ccc}
\hline & Recall & Precision \\
\hline Pixel Difference & 0.883 & 0.815 \\
\hline Histogram Difference & 0.774 & 0.724 \\
\hline Edge Count Difference & 0.712 & 0.745 \\
\hline Variance of Difference Histogram & 0.892 & 0.853 \\
\hline The Proposed Algorithm & 0.974 & 0.942 \\
\hline
\end{tabular}


paper, a new shot boundary detection and an R-frame selection algorithm that are essential for the automation of internal structure extraction are proposed.

On the contrary to the existing shot boundary detection algorithms, the proposed algorithm adopts dual detectors that are specifically designed for detecting cuts and gradual boundaries respectively. Also it has a delayed decision mechanism that not only arbitrates the results of the two detectors but also checks the validity of the detected shots, which resulted in a big improvements in overall accuracy.

Also for R-frame selection, the motion of the content is considered to select more meaningful frames. The adopted criteria for the decision is a changing pattern of pixel differences that are already calculated in the shot boundary detection, thus almost no additional processing time is required to solve the problem.

The experimental results show that the proposed algorithm is far more accurate in shot boundary detection than the compared traditional approaches. Also the selected R-frames are considered to be reasonable.

\section{REFERENCES}

Huang, L., Lee, J. C., Li, Q. and Xiong, W. (1996) An Experimental Video Database Management System Based On Advanced Object-Oriented Techniques: Proc. SPIE, Vol. 2670, pp.158-169.

Zhong, D., Zhang, H. J. and Chang, S. (1996) Clustering Methods for Video Browsing and Annotation: Proc. SPIE, Vol. 2670, pp.239-246.

Zhang., H. J., Low, C. Y., Smoliar, S. W. and Wu, J. H. (1995) Video Parsing, Retrieval and Browsing : An Integrated and Content-Based Solution : Proc. ACM Multimedia '95, pp. 15-24.

Ahanger, G. and Little, T. (1996) A Survey of Technologies for Parsing and Indexing Digital Video : Journal of Visual Communication and Image Representation, Special Issue on Digital Libraries, Vol. 7, No. 1, pp. 28-43.

Boreczky, J. S. and Rowe, L. A. (1996) Comparison of Video Shot Boundary Detection Techniques : IS\&T/SPIE, Vol. 2670, pp.170-179.

Zhang, H. J. and Smoliar, S. W. (1994) Developing Power Tools for Video Indexing and Retrieval : Proc. SPIE, Vol. 2185, pp. 140-149.

Ardizzone, E., Cascia, M. and Molinelli, D. (1996) Motion and Color Based Video Indexing and Retrieval : Int. Conf. on Pattern Recognition, ICPR, Wien, Austria. Liu, H. and Zick, G. L. (1995) Scene Decomposition of MPEG Compressed Video : Proc. SPIE, Vol. 2419, pp.26-37.

Meng, J. and Chang, S. (1996) Tools for Compressed-Domain Video Indexing and Editing : Proc. SPIE, Vol. 2670, pp. 180-191.

Zhang, A. and Multani, S. (1996) Implementation of Video Presentation in Database Systems : Proc. SPIE, Vol. 2670, pp.228-238.

Cascia, M. and Ardizzone, E. (1996) JACOB: Just a Content-Based Query System for Video Database : Proc. ICASSP-96, pp. 1-4

Picard, R. W. (1995) A Video Browser that Learns by Example : MIT Media 
Laboratory Technical Report \#383

Tou, J. T. and Gonzalez, R. C. (1974) Pattern Recognition Principles : AdditionWesley Publishing Company, Inc. pp.113-114.

\section{BIOGRAPHY}

Jae Yeon Lee received the $\mathrm{PhD}$ degree from Tokai University, Japan, in 1996. Se Yoon Chung received the MS degree from Inha University, Korea, in 1997. Byung Tae Chun received the MS degree from Soongsil University, Korea, in 1989.

Younglae J. Bae received the PhD degree from Kent University, U.K., in 1995.

All the authors are working for Systems Engineering Research Institute at the department of image processing. They are currently participating in the project for developing a video database which includes the following topics : shot boundary detection, content-based retrieval, caption recognition, etc. 